\title{
Molecular Dynamics Study of the Separation Behavior at the Interface between PVDF Binder and Copper Current Collector
}

\author{
Seungjun Lee \\ Department of Mechanical, Robotics and Energy Engineering, Dongguk University, Seoul 100715, Republic of Korea \\ Correspondence should be addressed to Seungjun Lee; sjunlee@dongguk.edu
}

Received 23 September 2015; Accepted 21 December 2015

Academic Editor: Miguel A. Correa-Duarte

Copyright (C) 2016 Seungjun Lee. This is an open access article distributed under the Creative Commons Attribution License, which permits unrestricted use, distribution, and reproduction in any medium, provided the original work is properly cited.

\begin{abstract}
In Li-ion batteries, the mechanical strengths at the interfaces of binder/particle and binder/current collector play an important role in maintaining the mechanical integrity of the composite electrode. In this work, the separation behaviors between polyvinylidene fluoride (PVDF) binders and copper current collectors are studied in the opening and sliding modes using molecular dynamics (MD) simulations. The simulation shows that the separation occurs inside the PVDF rather than at the interface due to the strong adhesion between PVDF and copper. This fracture behavior is different from the behavior of the PVDF/graphite basal plane that shows a clear separation at the interface. The results suggest that the adhesion strength of the PVDF/copper is stronger than that of the PVDF/graphite basal plane. The methodology used in MD simulation can directly evaluate the adhesion strength at the interfaces of various materials between binders, substrates, and particles at the atomic scales. The proposed method can therefore provide a guideline for the design of the electrode in order to enhance the mechanical integrity for better battery performance.
\end{abstract}

\section{Introduction}

Fracture of the electrode in Li-ion batteries has been ascribed as one of the main reasons for battery degradation [1-3]. As lithium ions move in and out of the active materials at the electrode, the active particles undergo volume changes, leading to a stress buildup. The intercalation induced stress eventually causes fracture and cracks of the electrode during charge/discharge cycles. The fracture results in isolation of active materials and loss of electric contact. Furthermore, the generation of new surfaces due to the crack enhances the solid electrolyte layer and dissolution of active materials. As a result, the mechanical failures increase side reactions such as impedance increase and capacity fade, eventually degrading the overall battery performance.

Since the electrode is composed of various materials including active particles, binders, carbon blacks, and current collectors, several scenarios are possible for fracture at the electrode: crack inside the particles, debonding of the particle and the binder, failure of the binder, and delamination of the particle/binder composite from the current collector. Fractures were experimentally observed at the electrode particles in Li-ion batteries and postulated as reasons for capacity fade $[4,5]$. Theoretical studies have also addressed fracture problems in terms of the effect of C-rates, particles sizes, and shapes $[3,6-8]$. These studies, however, focus on fractures that occur inside a particle, and less attention has been paid to interparticle fractures and debonding between particles and the current collector.

For fracture issues outside the particles, the adhesion strength of binders plays an important role for the mechanical integrity of the electrode composites, which significantly affects battery performance [9-11]. The importance of the binder becomes especially critical for high capacity materials such as Si [12] and Sn [13]. Hochgatterer et al. found that a chemical bond between binders and active particles improves cycling stability because the strong chemical interaction prevents severe mechanical swelling and maintains the shape of the electrode composite [14]. Komaba et al. reported that enhancing the adhesion strength of a binder in the electrode composite can reduce the capacity retention because it suppresses the particle isolation and detachment of the electrode film from the current collector [15]. 
Recently, Chen et al. studied the adhesion strength between particles and between electrode films and current collectors using coupled microscratch and digital image correlation techniques [10]. Although the study found the relative strength of the particle cohesion and the film/current collector adhesion, the qualitative strengths are provided based on indirect measurements. Indeed, it is difficult to directly measure the quantitative strength of these systems at small scale by experiment. Moreover, although the microscratch test can qualitatively estimate the strength of a similar order of microparticles using a micrometers tip, the test should be able to measure the strength at nanoscales for more precise evaluation. For example, for the particle/particle decohesion, it is difficult to clearly observe whether the separation occurs in the middle of the binder or at the interface between the binder and the particle by experiment. Therefore, atomic simulations are useful to evaluate the mechanical strength of such a small system. Molecular dynamics simulations have been widely used to study the separation behavior at the various interfaces such as metal/metal $[16,17]$, metal/ceramic [18], metal/metal oxide [19], carbon nanotube/polymer [20, 21], and graphene/polymer [22]. Recently, the separation responses of the interface between the graphite particle and the PVDF binder in Li-ion batteries are studied using MD simulations [23]. The obtained traction-separation responses from the simulation will be used for the input data of the cohesive zone model at continuum scales.

This work investigates the separation behavior at the interface of the binder and the current collector. Polyvinylidene fluoride (PVDF) and copper $(\mathrm{Cu})$ are selected for the binder and the current collector materials, respectively, which are commonly used in current commercial batteries. The PVDF/copper interface is modeled at the atomic level, and the normal and shear loading are applied to the system to study the separation responses in the opening and the sliding mode. The mechanical behavior of the PVDF/copper interface is compared with that of the PVDF/graphite [23], where the PVDF is attached to the basal plane of graphite. From the MD simulations, it is found that the adhesion strength of the PVDF/copper is stronger than that of the PVDF/graphite basal plane. The results indicate that the adhesion strength of the PVDF on the graphite basal plane is weak and an interparticle fracture therefore potentially initiates from the PVDF/graphite interface.

\section{Simulation Method}

2.1. Atomic Potentials. The atomic interactions in copper are described using an embedded-atom method (EAM) potential [24]. The total energy $E_{i}$ of atom $i$ is expressed as

$$
E_{i}=F_{i}\left(\rho_{i}\right)+\frac{1}{2} \sum_{j \neq i} \phi_{i j}\left(r_{i j}\right),
$$

where $F_{i}(\rho)$ is the embedding energy of atom $i$ into the background electron density, which is a function of the electron density $\rho_{i}$ at atom $i$. The electron density is approximated by the superposition of electron densities, $\rho_{i}=$ $\sum_{j \neq i} \rho_{j}\left(r_{i j}\right)$, where $\rho_{j}$ is the electron density contributed by atom $j$ separated by distance $r_{i j} . \phi_{i j}\left(r_{i j}\right)$ is the core-core pair repulsion between $i$ and $j$ atoms.

The atomic interactions in the PVDF binder are described by the potential functions developed by Byutner and Smith [25]. The van der Waals and electrostatic interactions are considered for the nonbonding interaction and the stretching, angle bending, and torsional terms are considered for the covalent interaction. The potential was validated in the previous study [23] by calculating the density of the PVDF.

For the interaction between PVDF and copper, the metal/polymer interface may involve various adhesion mechanisms including physical adsorption, chemical adsorption, mechanical interlocking, polymer diffusion, and weak boundary layer [26]. In the study, it is assumed that no modification is made on the inert copper material; thus the polymer is bonded with copper by physical interactions without chemical bonds. In the case, van der Waals interactions can properly describe the pair interactions [27]. The van der Waals interaction is described with a Lennard-Johns (LJ) form written as $E\left(r_{i j}\right)=4 \varepsilon_{i j}\left[\left(\sigma_{i j} / r_{i j}\right)^{12}-\left(\sigma_{i j} / r_{i j}\right)^{6}\right]$. The parameters of the LJ potential are calculated by the LorentzBerthelot mixing rule. The values of sigma and epsilon are calculated using an arithmetic mean of $\sigma_{i j}=\left(\sigma_{i i}+\sigma_{j j}\right) / 2$ and a geometric mean of $\varepsilon_{i j}=\sqrt{\varepsilon_{i i} \varepsilon_{j j}}$, respectively. Although the mixing rule is simple, it is widely used to describe nonbonding interactions of the van der Waals term, especially for the interactions with polymers. It was successfully implemented for the separation behavior of the various interfaces including polymer/graphene $[20,22]$ and polymer/carbon nanotube $[21,28]$. It also has been effectively used for the material pair with very different nature such as polymer/metal systems. The examples are epoxy/copper [27, 29], MMA polymer/metal oxides $\left(\mathrm{Al}_{2} \mathrm{O}_{3}, \mathrm{Fe}_{2} \mathrm{O}_{3}, \mathrm{SiO}_{2}\right.$, and $\left.\mathrm{TiO}_{2}\right)$ [30], and ABS polymer/copper [31]. These studies used the COMPASS force field [32], which describes the pair interaction of different species using a mixing rule. To use the mixing rule, the Buckingham potentials for $-\mathrm{C},-\mathrm{F}$, and $-\mathrm{H}$ atoms in the PVDF are fitted to the LJ form to obtain the LJ parameters for the PVDF binder. The values of the LJ potential for copper are used with $\varepsilon_{\mathrm{Cu}-\mathrm{Cu}}=0.415 \mathrm{eV}$ and $\sigma_{\mathrm{Cu}-\mathrm{Cu}}=2.277 \AA[33,34]$. The parameters used in simulation are summarized in Table 1.

For the validation of the pair potentials, the work of separation of the PVDF/copper is compared with a similar system of the polymer/copper, since the adhesion strength of the $\mathrm{PVDF} / \mathrm{copper}$ is limited in literature. The work of separation is calculated by the following equation using a slab model [23]:

$$
\begin{aligned}
& W_{\text {sep }} \\
& \quad=\left(E_{\mathrm{PVDF}}+E_{\text {copper }}-E_{\mathrm{PVDF} / \text { copper }}\right) / A,
\end{aligned}
$$

where $E_{\mathrm{PVDF}}, E_{\text {copper }}$, and $E_{\mathrm{PVDF} / \text { copper }}$ are the total energy of the PVDF, copper, and PVDF/copper slab, respectively, and $A$ is the interfacial area. The total energy of the slab models is calculated as $E_{\mathrm{PVDF}}=2675.9 \mathrm{eV}, E_{\text {copper }}=-7492.3 \mathrm{eV}$ and $E_{\mathrm{PVDF} / \text { copper }}=-4859.2 \mathrm{eV}$, and the interfacial area $A$ is $1296 \AA^{2}$. Using the values, the work of separation $W_{\text {sep }}$ is calculated as $0.53 \mathrm{~J} / \mathrm{m}^{2}$. The calculated value is similar to 
TABLE 1: LJ parameters used for interactions between PVDF and copper

\begin{tabular}{lccc}
\hline $\begin{array}{l}\text { Atom } i \text { in } \\
\text { PVDF }\end{array}$ & $\begin{array}{c}\text { Atom } j \text { in } \\
\text { copper }\end{array}$ & $\varepsilon_{i j}(\mathrm{eV})$ & $\sigma_{i j}(\AA)$ \\
\hline $\mathrm{H}$ & $\mathrm{Cu}$ & 0.0133 & 2.625 \\
$\mathrm{~F}$ & $\mathrm{Cu}$ & 0.0276 & 2.66 \\
$\mathrm{C}$ & $\mathrm{Cu}$ & 0.0413 & 2.835 \\
\hline
\end{tabular}

TABLE 2: Cases of different system sizes in MD simulations.

\begin{tabular}{lccc}
\hline Case & $\begin{array}{c}\text { Number of } \\
\text { PVDF chains }\end{array}$ & $\begin{array}{c}\text { Size of one } \\
\text { side }(\AA)\end{array}$ & $\begin{array}{c}\text { Area of copper } \\
\text { substrate }\left(\AA^{2}\right)\end{array}$ \\
\hline 1 & 10 & 25.0 & 625.0 \\
2 & 20 & 28.8 & 829.4 \\
3 & 30 & 36.0 & 1296.0 \\
4 & 40 & 39.7 & 1576.1 \\
\hline
\end{tabular}

the reported value of $0.51 \mathrm{~J} / \mathrm{m}^{2}$ for the interface between acrylonitrile-butadiene-styrene (ABS) and copper [31], which indicates that the pair potentials are reasonable.

2.2. MD Model. A representative model for the MD simulations is shown in Figure 1. In the figure, blue is hydrogen, green is fluoride, orange is carbon, and pink is copper. The PVDF chains are composed of 20 repeating units - $\left[\mathrm{CH}_{2}-\right.$ $\mathrm{CF}_{2}$ ]- and each end is terminated by $-\mathrm{CH}_{3}$ and $-\mathrm{CF}_{3}$. The simulations are performed for four different simulation sizes in order to check the size effect on simulation results and obtain reliable results. The system size is determined by the number of the PVDF chains, as summarized in Table 2. The same molecular weight of $1365 \mathrm{~g} / \mathrm{mol}$ is used as that in the previous simulations of PVDF/graphite [23] to compare the separation behavior at the same condition. The periodic boundary condition is used in the $x$ and $y$ direction. A large height of the simulation box, which is $150 \AA$, is given in order to prevent interactions in the $z$ direction. The top of the PVDF and the bottom of the copper are fixed at $25 \%$ to serve as boundaries. The displacements of the top rigid region in the PVDF are increased vertically and horizontally with a constant velocity of $0.2 \AA / p s$ for the tensile and shear loading, respectively.

2.3. MD Simulation. All MD simulations are performed by LAMMPS. A timestep of 1 femtosecond is used. The NoseHoover thermostat and the Nose-Hoover barostat are used. The simulations are performed in three steps. At the first step, only the PVDF chains that are aligned in the $z$ direction are introduced in the simulation box. The chains are relaxed to remove the artificial initial alignment for 100 picoseconds. During the simulation, the chains are agglomerated due to the cohesion of the PVDF. At the second step, a copper slab with a thickness of $20 \AA$ is inserted below the PVDF chains. As the system is heated to $525 \mathrm{~K}$, which is above the melting point of PVDF, the chains are attracted to the copper surface due to the adhesive interactions. After 100 picoseconds, the system is cooled to $300 \mathrm{~K}$ and relaxed over 1 nanosecond until the system is equilibrated. The NVT ensemble is used during the annealing process and the NPT ensemble with a pressure of

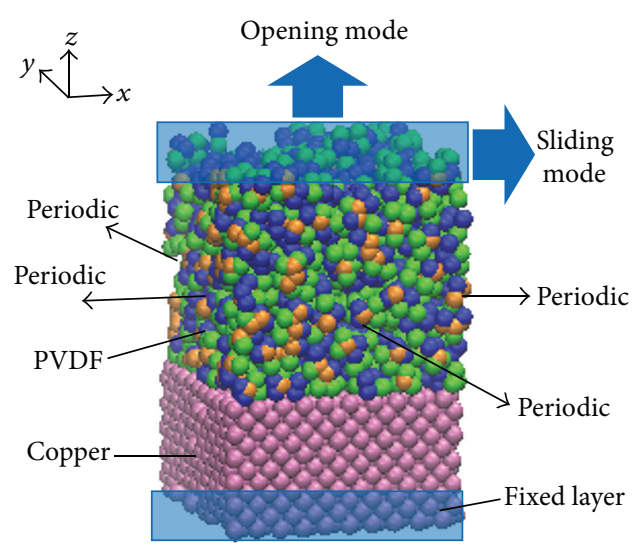

FIGURE 1: Representative model for the MD simulations.

1 bar is used during the relaxation process. At the third step, tensile loading is applied for the opening mode by increasing the displacements of the rigid region in the $z$ direction and the normal stress of the unfixed region is calculated as a function of strains. The simulation is performed until the system separates into two parts. A shear loading is also applied to the system for the sliding mode.

The stress is calculated in the form of the virial stress [35, 36]. Since the PVDF binder undergoes a large deformation and irregular voids are created, the atomic volume of the PVDF binder is calculated using the sphere-probing method [23]. Small spheres are filled in the system and the spheres overlapped with the PVDF atoms are checked. The volume of the checked spheres is summed to represent the volume of the deformed PVDF.

\section{Results and Discussion}

3.1. Opening Mode. Figure 2 shows the traction-separation responses for 10 (black) and 20 (red) PVDF chains. The stresses are averaged every 1 picosecond to reduce thermal fluctuations. The curves show large fluctuations, especially for 10 chains, due to the small system size. However, they follow a typical trend of the traction-separation response. As the normal strain increases, the normal stress increases and reaches the maximum. The stress then decreases gradually and converges to zero. Compared to the previous results of the PVDF/graphite interface [23], the curve has a wider distribution around the maximum stress and the shape of the curve is rounder. The maximum stress reaches about $0.4 \mathrm{GPa}$ and $0.45 \mathrm{GPa}$ for 10 chains and 20 chains, respectively. The separation displacement is around $15 \AA$ when the maximum stress occurs, and the entire separation occurs around $45 \AA$.

The MD simulations capture the detailed fracture process at the atomic scale. Representative snapshots of 10 chains are shown in Figure 3. As the normal strains are applied, small voids quickly appear on the PVDF. The stress drop shown in Figure 2 around the separation displacements of $5 \sim 10 \AA$ for 10 chains is due to the separation among the PVDF chains. The separation is caused by the weak interaction of the van der Waals interactions acting between the chains. The effect of the weak binding strength is relatively large 


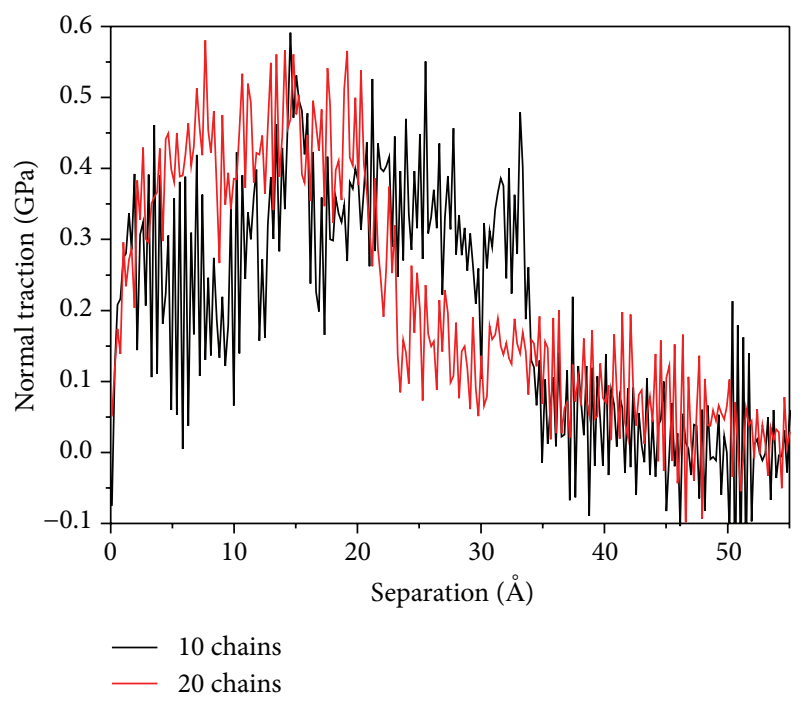

FIGURE 2: Traction-separation responses for the cases of 10 chains (black) and 20 chains (red).

for the smaller systems. After the reduction in the normal traction, the stress increases again and the stress level exceeds $0.3 \mathrm{GPa}$. During the increase of the normal traction around the separation displacement of 10 15 $\AA$, the PVDF chains are stretched upward (Figure 3(c)). As the separation increases further, the void increases and the column becomes thinner (Figure 3(d)). At a separation of around $40 \AA$, a long and thin PVDF column emerges, connecting the top and bottom groups of the PVDF (Figure 3(e)). Finally, the column breaks in the middle at a separation of $45 \AA$ (Figure $3(\mathrm{f})$ ). As the disconnected chains in the column are attracted to the top and bottom groups, the column disappears. At the end of the simulation, the PVDF binder is separated by almost half.

Figure 4 shows the traction-separation responses of 30 (green) and 40 (blue) chains. Compared to the small systems, the large system has a sharp peak in the curve. For 30 chains, the maximum stress is about $0.4 \mathrm{GPa}$ at a separation of $10 \AA$, and for 40 chains the maximum stress is about $0.35 \mathrm{GPa}$ at a separation of $5 \AA$. The maximum separation of the larger systems is around $55 \AA$, which is greater than that of the smaller systems.

Snapshots of the MD simulation for 30 chains are shown in Figure 5. Initially, as the normal strain increases, the stress increases almost linearly, showing elastic deformation. After a separation of $3 \AA$, small voids are observed almost everywhere in the PVDF (Figure 5(a)). The slope of the curve decreases after the small voids appear. As the voids enlarge, a hole emerges in the PVDF and the normal stress begins to decrease. The stress continuously decreases as the hole enlarges. Some chains are aligned vertically and generate a wall in the middle as they are stretched upward (Figure 5(b)). As strains increase further, a hole appears on the PVDF wall (Figure 5(c)). The hole enlarges, and eventually the binder is separated in the middle (Figure 5(d)).

For all the cases, the separation occurs in the middle of the PVDF. At the end of the simulations, half of the PVDF remains on the copper surface and the other half is attached to the top boundary. The simulation results indicate that the mechanical strength between the PVDF and copper is stronger than that inside the PVDF. This separation behavior in the PVDF/copper system significantly differs from the separation behavior in the PVDF/graphite system presented in the previous study [23]. For the PVDF/graphite, the separation occurs near the interface for the small systems or clearly at the interface for the large systems. However, for the PVDF/copper, the separation occurs in the middle of the PVDF chains for all cases, where the traction-separation responses show a ductile behavior because the separation occurs inside the polymer. Both the maximum stress and the maximum separation for the PVDF/copper are larger than those for the PVDF/graphite. This means that the PVDF/copper, which has a larger area under the curve, needs more energy for the separation at the interface.

To confirm the different separation behaviors, the evolution of 1 PVDF chain that is attached to the substrate is tracked during the simulation. Figures 6 and 7 show the MD snapshots of a PVDF chain on the surface of copper and graphite, respectively. For the graphite case in Figure 6, the attached part of the chain on the surface translates as the upper part of the chain is stretched (Figure 6(b)). Although the chain does not belong to the top rigid region for the applied normal loading, the upper part of the chain is pulled upward due to the cohesion strength of other chains. As the stretching increases further, the bottom part gradually separates from the surface (Figure 6(c)) and the entire chain is eventually separated, moving upward together with other PVDF chains (Figure 6(c)). Thus, it shows a separation at the interface between the PVDF and graphite. On the other hand, for the copper case in Figure 7, the attached bottom part is adhered to the surface and does not move when the upper part is stretched upward (Figure $7(\mathrm{~b})$ ). As the stretching increases, the chain is separated from the group of the PVDF chains and left on the surface (Figure 7(c)). After separation occurs in the middle of the PVDF, the upper part of the chain loses the pulling strength from the top PVDF group and returns to the unstretched state (Figure 7(d)). The location of the chain does not change from its original position during the entire simulation. In the simulation of the PVDF/copper, the strong interaction between the PVDF and copper prevents the PVDF chains near the interface from deforming flexibly upon the load. Therefore, the bottom part of the PVDF binder acts as a fixed boundary, similar to the top fixed layer when external loading is applied to the system. Thus, two opposing forces are applied to the binder and the failure occurs in the middle of the binder.

Since it is impossible to directly compare the quantitative strength at the interfaces between the PVDF/copper and the $\mathrm{PVDF} /$ graphite due to their different separation behaviors, for both the cases, all the PVDF binder is fixed and strained in the $z$ direction in order to apply the same boundary condition. In this way, the separation occurs at the interface regardless of the substrate material. The normal stress is calculated for the unfixed part of the copper and graphite and is shown in Figure 8. The maximum stress of the PVDF/copper is calculated as $4.2 \mathrm{GPa}$ and that of the PVDF/graphite is calculated as $0.6 \mathrm{GPa}$, showing a difference of seven times. 


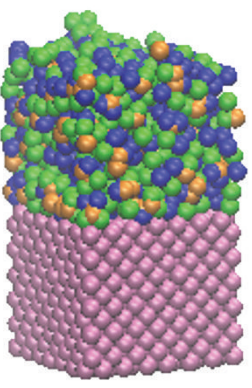

(a)

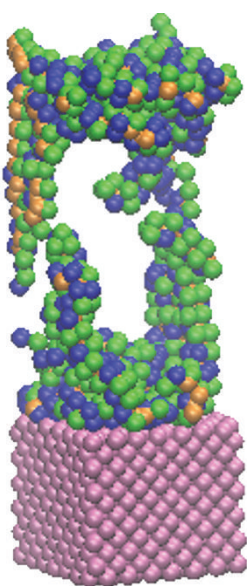

(d)

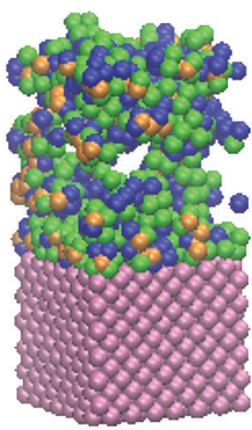

(b)

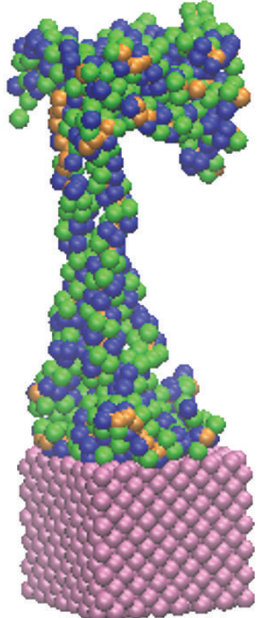

(e)

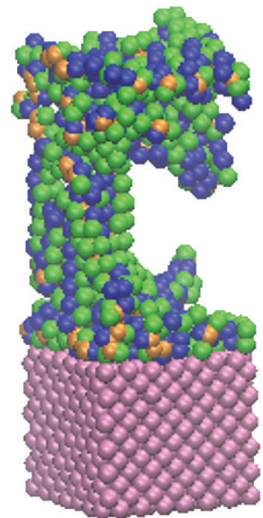

(c)
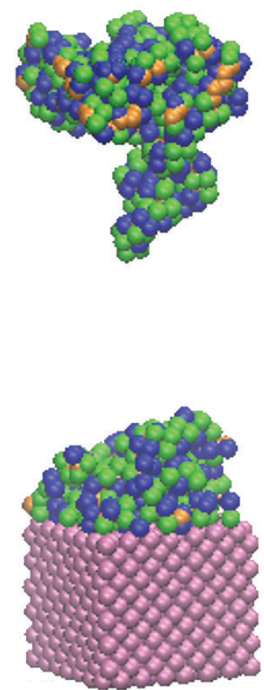

(f)

FIgURE 3: Snapshots of the MD simulation for 10 chains at various strains of (a) $0 \AA$, (b) $6 \AA$, (c) $15 \AA$, (d) $31 \AA$, (e) $41 \AA$, and (f) $48 \AA$.



FIgURE 4: The traction-separation responses for the cases of 30 chains (green) and 40 chains (blue). 


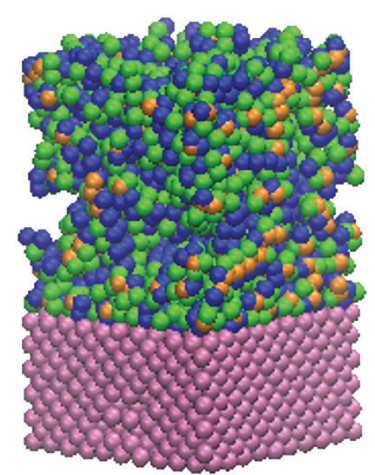

(a)

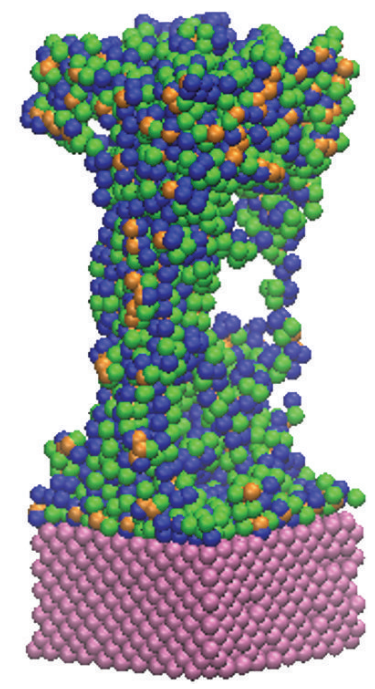

(c)



(b)

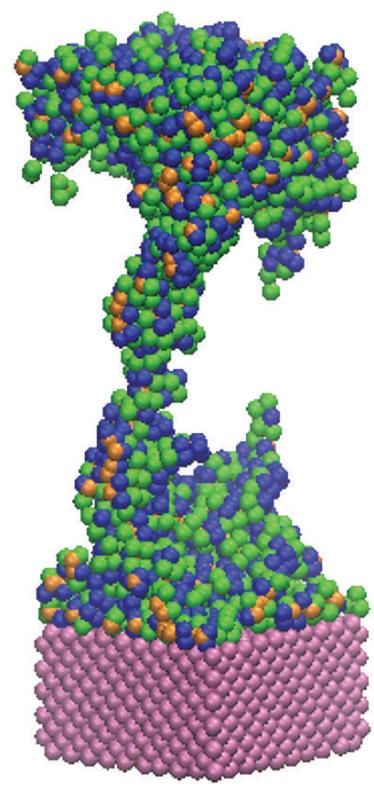

(d)

FIgURE 5: Snapshots of the MD simulation of 30 chains at various separations of (a) $6 \AA$, (b) $27 \AA$, (c) $37 \AA$, and (d) $54 \AA$.

The work of separation is calculated as $0.73 \mathrm{~N} / \mathrm{m}$ for the $\mathrm{PVDF} /$ copper and $0.09 \mathrm{~N} / \mathrm{m}$ for the $\mathrm{PVDF} /$ graphite.

The stronger adhesion of the PVDF/copper than the $\mathrm{PVDF} /$ graphite is mainly due to the larger well depth of the LJ potential for the substrate. For copper, the value of epsilon is $0.415 \mathrm{eV}$, and for graphite the value of epsilon is $0.00284 \mathrm{eV}$. The interactions between the PVDF and the substrate are calculated by the mixing rule so the large potential depth of the substrate results in the strong adhesion between the substrate and the binder. The pair potentials for the $\mathrm{C}$ (PVDF)-Cu (current collector) and the $\mathrm{C}$ (PVDF)-C (graphite) are shown in Figure 9 and the difference of the potential well depths is almost one order of magnitude. Since the pair potentials are defined by the mixing rule, the LJ parameters for copper are also important. In literature, the potential well depth for copper lies in the range of 0.17 $0.42 \mathrm{eV}[33,34,37-40]$. Since the value of epsilon used in the study is $\varepsilon_{\mathrm{Cu}-\mathrm{Cu}}=0.415 \mathrm{eV}$, a case of 20 chains is tested using a relatively small value of $\varepsilon_{\mathrm{Cu}-\mathrm{Cu}}=0.167 \mathrm{eV}$ among the available reference. Although the pair potential well depths between $C$, $\mathrm{H}$, and $\mathrm{F}$ (PVDF) and $\mathrm{Cu}$ are reduced almost half, the similar separation behaviors are observed: the separation occurs in the middle of the PVDF and the PVDF chains that directly contact the copper surface do not move on the surface. In addition, the contact angle of the melted PVDF on the copper surface is measured after it cools down. The measured angle is approximately $3^{\circ}$, which is almost flat. Compared to the contact angle of about $30^{\circ}$ for the PVDF/graphite [23], the pair interactions of the $\mathrm{PVDF} /$ copper are stronger than those of the PVDF/graphite. 


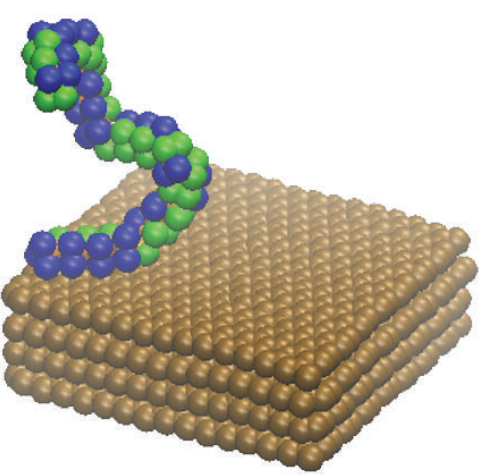

(a)

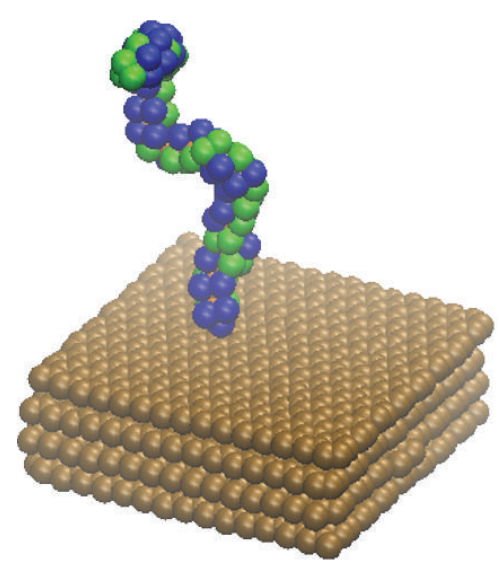

(c)

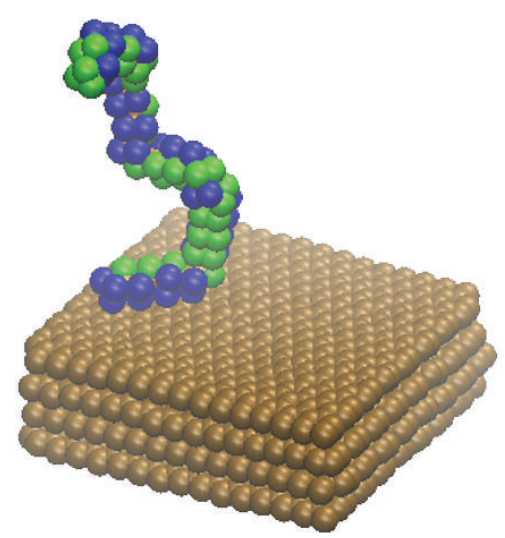

(b)

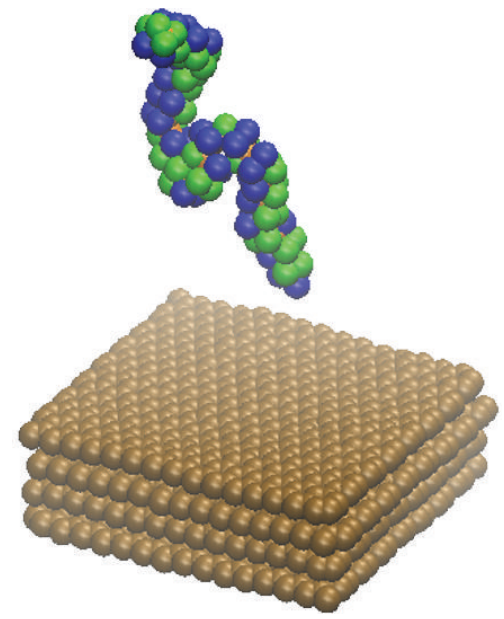

(d)

Figure 6: Snapshots of one PVDF chain in the PVDF/graphite system during the normal separation simulation.

The result, however, differs from that in the recent study, which reported that the adhesion of carbon particle/copper is weaker than the adhesion of carbon particle/PVDF [10]. The main reason for the different results is that the graphite basal plane is considered for the simulation model. It is possible that the PVDF chemically bonds with the graphite due to the dangling bonds on the edge plane of graphite so the adhesion strength may be significantly strong between the PVDF and the edge plane. Although the simulations did not consider the separation of PVDF from the edge plane, the simulation results suggest that the adhesion of the PVDF/basal plane is weak, whereby a crack may initiate on the basal plane rather than between the PVDF and the copper current collector. Therefore, reducing the area of the basal plane in graphite can help to increase the mechanical binding strength at the electrode.

3.2. Sliding Mode. In the sliding mode, the shear stress $\left(\sigma_{x z}\right)$ is calculated while the fixed top layer is moved in the $x$ direction. First, the entire PVDF is fixed and moved horizontally to validate the sliding simulation. It is expected that the response shows a periodic pattern because it should reflect the periodicity of the copper lattice. The calculated shear stress is shown in Figure 10(a). The shear response shows a periodic trend and the wavelength matches the copper lattice unit cell of $3.6 \AA$.

Figure 10(b) shows the shear response for a representative case of 30 chains in the sliding mode with the top part of the PVDF fixed. The shear response shows a similar trend to the normal traction-separation response. Initially, the shear stress increases almost linearly. The stress then reaches the maximum of $0.35 \mathrm{GPa}$ at a shear displacement of $13 \AA$. After the peak, the stress gradually decreases. After a shear displacement of $120 \AA$, the shear stress fluctuates almost constantly with an average of $0.1 \mathrm{GPa}$.

In the sliding mode, the stress response of the PVDF/copper significantly differs from that of the PVDF/graphite. The shear response of the PVDF/graphite oscillates because the PVDF is separated at the interface due to the weak interaction and shows a periodic response similar to the case in which all PVDF are fixed. For the PVDF/copper, however, the interaction between the PVDF and copper is strong so the separation occurs in the middle of the PVDF rather than at the PVDF/copper interface.

Figures 11 and 12 show representative snapshots of two chains in the PVDF that behave differently during the sliding 


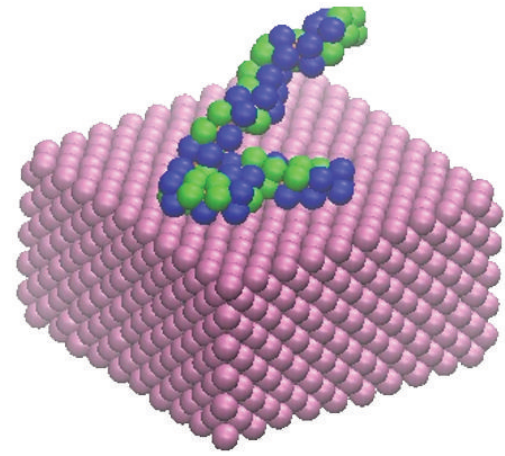

(a)

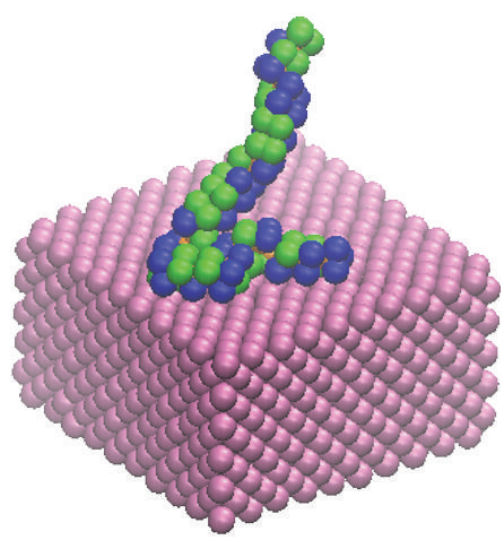

(c)

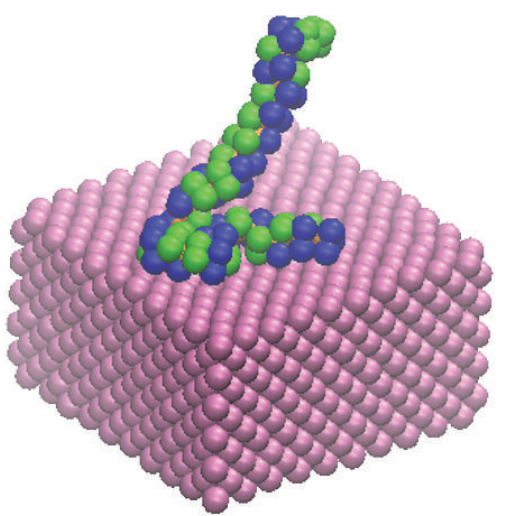

(b)

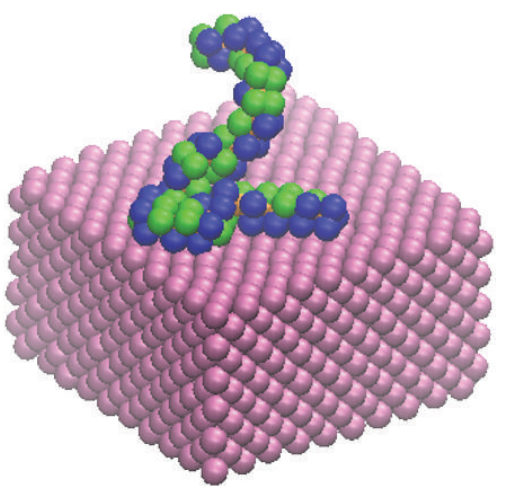

(d)

FIGURE 7: Snapshots of one PVDF chain in the PVDF/copper system during the normal separation simulation.

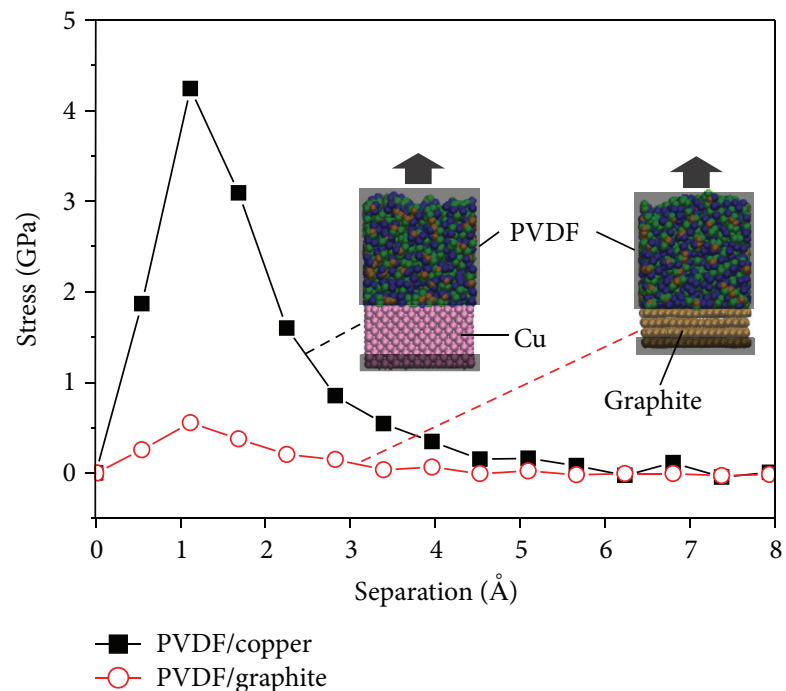

FIgURE 8: Comparison of the traction-separation response at the interface of the PVDF/copper and the PVDF/graphite for the cases in which all the PVDF atoms are fixed.

simulation of the PVDF/copper. The chain shown in Figure 11 slides on the copper surface as the shear displacements are

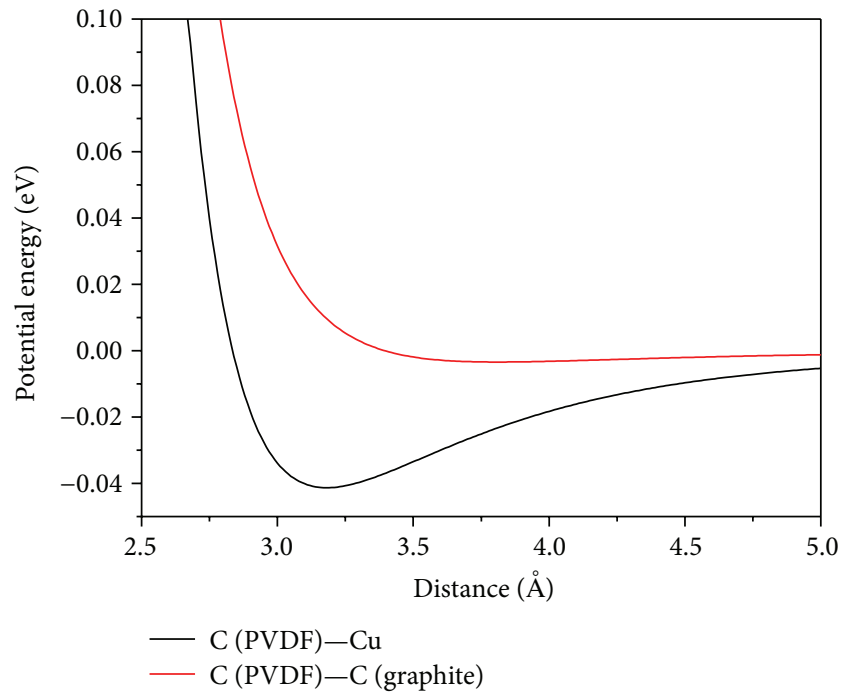

Figure 9: Pair potentials for the C (PVDF)-Cu and the C (PVDF)C (Graphite).

applied (Figure 11(b)). However, after a shear displacement of $100 \AA$, the chain stops moving. Although the top part of the chain is stretched to the $x$ direction, the bottom part is 


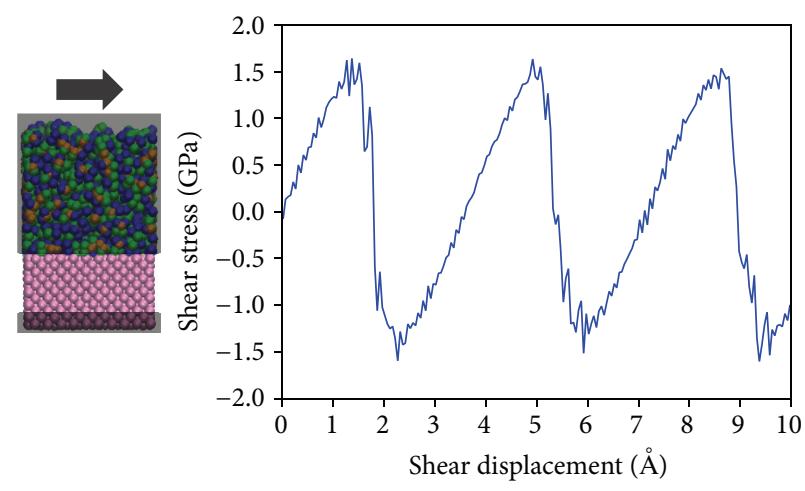

(a)
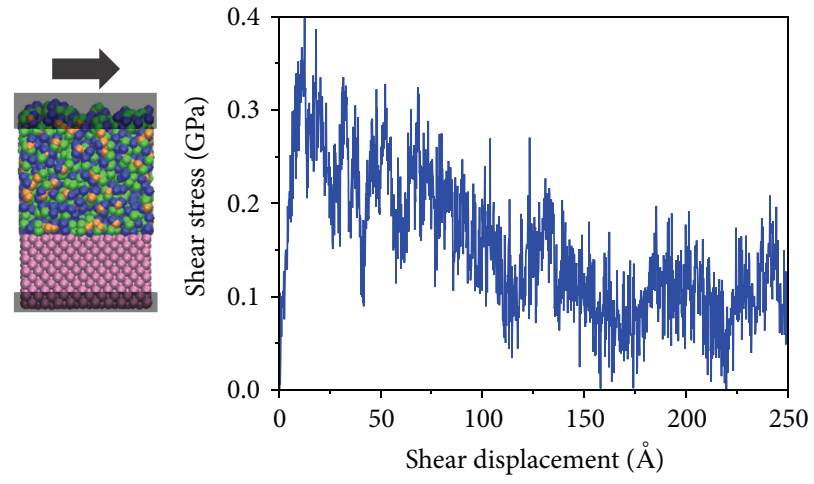

(b)

FIGURE 10: Shear stress responses in the sliding mode for the cases in which (a) all of the PVDF is fixed and (b) only the top PVDF atoms are fixed.

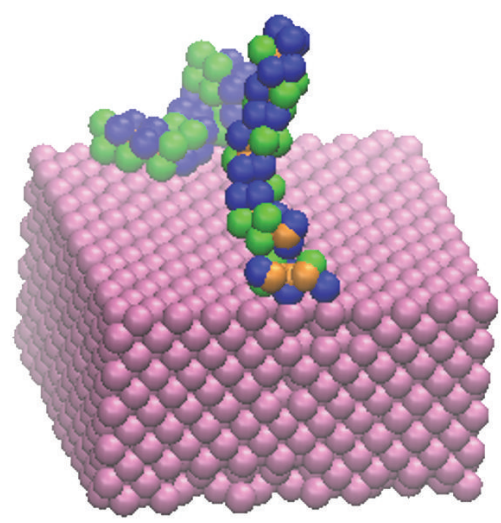

(a)



(c)

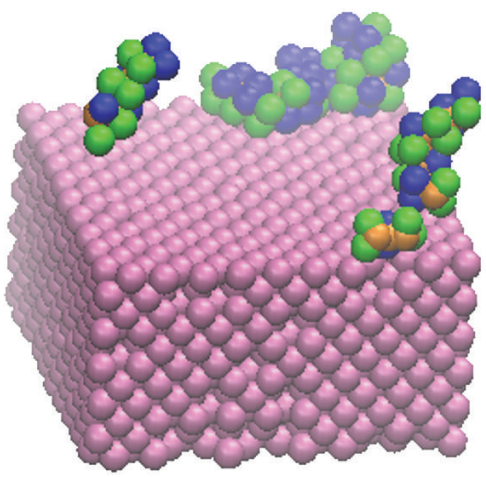

(b)

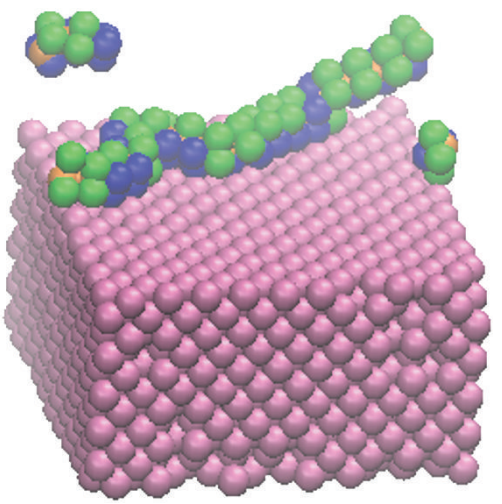

(d)

FIGURE 11: Snapshots of one PVDF chain at the bottom part in the sliding motion at a shear displacement of (a) $0 \AA$, (b) $35 \AA$, (c) $100 \AA$, and (d) $250 \AA$.

adhered to the substrate and does not move. On the other hand, the chain in Figure 12 behaves differently. Initially, the chain moves on the surface (Figure 12(b)). However, in the middle of the simulation, the entire chain is detached from the surface and lies almost horizontally in the $x$ direction (Figure 12(c)). The chain then continuously translates until the end of the simulation, keeping the horizontally stretched manner (Figure 12(d)). From the snapshots, we can confirm that, in the sliding mode, the PVDF is separated in the middle, as shown similarly in the opening mode. Since each chain in Figures 11 and 12 belongs to the top and bottom of the PVDF, respectively, the bottom chain is fixed and the top chain moves along with the sliding strains. The reason that the shear stress is converged to a certain level, not to zero, is 


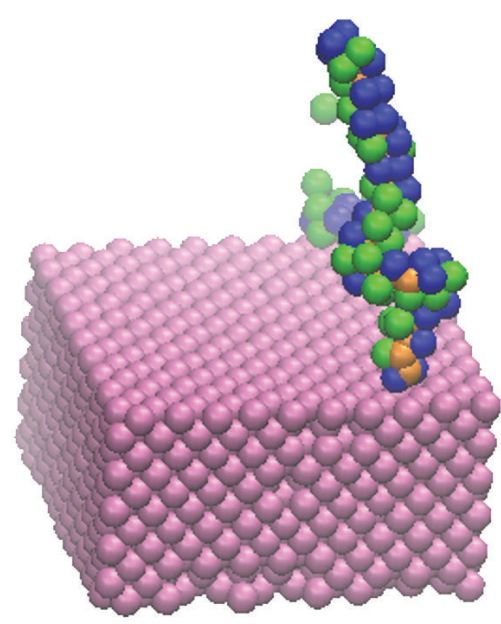

(a)

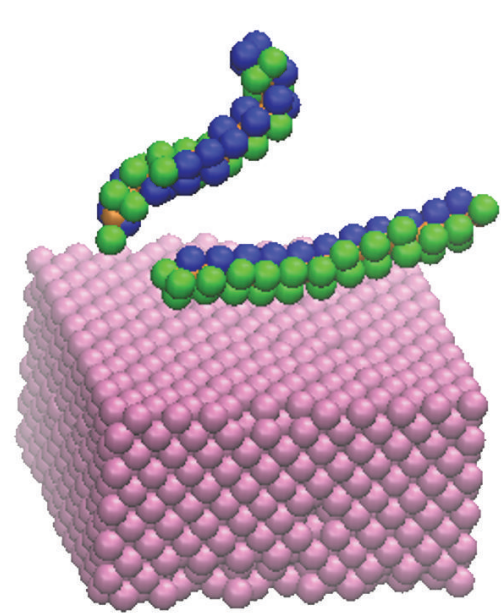

(c)

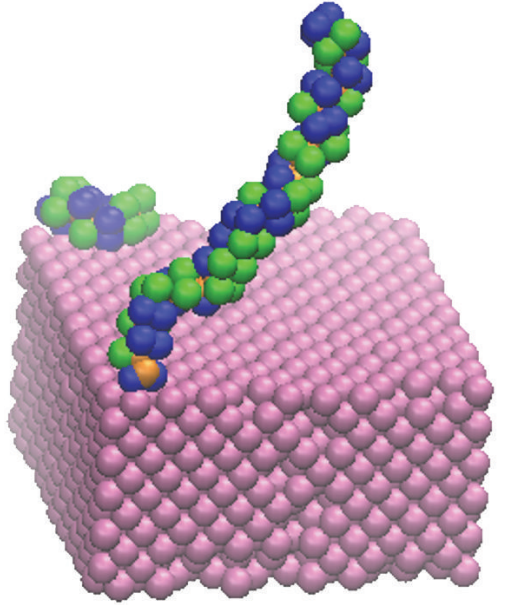

(b)

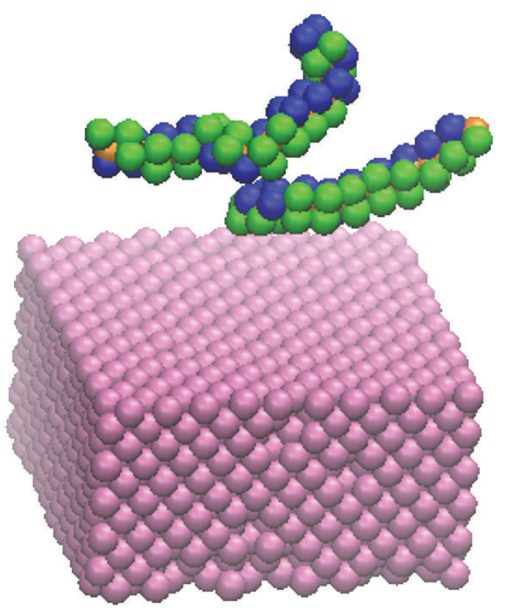

(d)

FIGURE 12: Snapshots of one PVDF chain at the top part in the sliding motion at a shear displacement of (a) $0 \AA$, (b) $35 \AA$, (c) $100 \AA$, and (d) $250 \AA$. .

that the simulation is performed in the periodic conditions so a new surface is not generated. The averaged shear stress that fluctuates at the end of the simulation is due to the sliding motion between two separated PVDF groups.

\section{Conclusions}

In this study, MD simulations were performed to investigate the separation responses at the interface of the PVDF binder and the copper current collector in the opening and sliding mode. In the opening mode, the maximum normal traction is calculated at around $0.35 \sim 0.45 \mathrm{GPa}$ and the maximum normal separation is $45 \sim 55 \AA$ at different system sizes. In addition to the quantitative information, the simulations at all the cases show that the separation occurs in the middle of the PVDF binder rather than at the interface between the PVDF and the copper substrate due to strong adhesion of the $\mathrm{PVDF} /$ copper. The strong adhesion also results in the similar stress response of the sliding mode to that of the opening mode. Compared to the separation behavior of the PVDF on the graphite basal plane, the simulation reveals that the adhesion strength of the PVDF/copper is greater than that of the PVDF/graphite basal plane. Although the adhesion of the binder on the edge plane of the graphite needs to be further evaluated in order to rank the adhesion strength between particles/binders and binders/current collectors, it is clear that the adhesion strength of the PVDF on the graphite basal plane is weaker than that of the PVDF/copper; thus, the crack initiation at the interface of the binder on the graphite basal plane is critical for the fracture of the electrode in the Li-ion batteries.

Although it is found that the adhesion of the PVDF/ copper is relatively strong, the high stress created during charge/discharge cycles may cause fracture at the interface. The fracture results in loss of electric contact between the electrode composite and the current collector, leading to resistance increase and capacity fade. The study reveals that the stress level for fracture at the PVDF/copper interface and the obtained data can be used for inputs of the continuum model in order to investigate fracture behavior of the 
electrode system and the relationship between the fracture and the degradation of Li-ion batteries. The framework in this work can be used for the different kinds of binders and potentially proposes new binder, which enhances the mechanical integrity of the electrode.

\section{Conflict of Interests}

The author declares that there is no conflict of interests regarding the publication of this paper.

\section{Acknowledgment}

This research was supported by the Basic Science Research Program, through the National Research Foundation of Korea (NRF), funded by the Ministry of Education (NRF2015R1D1A1A01057759).

\section{References}

[1] R. A. Huggins and W. D. Nix, "Decrepitation model for capacity loss during cycling of alloys in rechargeable electrochemical systems," Ionics, vol. 6, no. 1-2, pp. 57-63, 2000.

[2] H.-L. Zhang, F. Li, C. Liu, J. Tan, and H.-M. Cheng, "New insight into the solid electrolyte interphase with use of a focused ion beam," The Journal of Physical Chemistry B, vol. 109, no. 47, pp. 22205-22211, 2005.

[3] M. Zhu, J. Park, and A. M. Sastry, "Fracture analysis of the cathode in li-ion batteries: a simulation study," Journal of the Electrochemical Society, vol. 159, no. 4, pp. A492-A498, 2012.

[4] H. Wang, Y.-I. I. Jang, B. Huang, D. R. Sadoway, and Y.-M. Chiang, "Tem study of electrochemical cycling-induced damage and disorder in $\mathrm{LiCoO}_{2}$ cathodes for rechargeable lithium batteries," Journal of the Electrochemical Society, vol. 146, no. 2, pp. 473-480, 1999.

[5] D. Wang, X. Wu, Z. Wang, and L. Chen, "Cracking causing cyclic instability of $\mathrm{LiFePO}_{4}$ cathode material," Journal of Power Sources, vol. 140, no. 1, pp. 125-128, 2005.

[6] W. H. Woodford, Y.-M. Chiang, and W. C. Carter, "Electrochemical shock' of intercalation electrodes: a fracture mechanics analysis," Journal of the Electrochemical Society, vol. 157, no. 10, pp. A1052-A1059, 2010.

[7] K. Zhao, M. Pharr, J. J. Vlassak, and Z. Suo, "Fracture of electrodes in lithium-ion batteries caused by fast charging," Journal of Applied Physics, vol. 108, no. 7, Article ID 073517, 2010.

[8] T. K. Bhandakkar and H. Gao, "Cohesive modeling of crack nucleation in a cylindrical electrode under axisymmetric diffusion induced stresses," International Journal of Solids and Structures, vol. 48, no. 16-17, pp. 2304-2309, 2011.

[9] N. Yuca, H. Zhao, X. Song et al., "A systematic investigation of polymer binder flexibility on the electrode performance of lithium-ion batteries," ACS Applied Materials \& Interfaces, vol. 6, no. 19, pp. 17111-17118, 2014.

[10] J. Chen, J. Liu, Y. Qi, T. Sun, and X. Li, "Unveiling the roles of binder in the mechanical integrity of electrodes for lithium-ion batteries," Journal of the Electrochemical Society, vol. 160, no. 9, pp. A1502-A1509, 2013.

[11] M. Wu, X. Xiao, N. Vukmirovic et al., "Toward an ideal polymer binder design for high-capacity battery anodes," Journal of the American Chemical Society, vol. 135, no. 32, pp. 12048-12056, 2013.
[12] A. Magasinski, B. Zdyrko, I. Kovalenko et al., "Toward efficient binders for Li-ion battery Si-based anodes: polyacrylic acid," ACS Applied Materials \& Interfaces, vol. 2, no. 11, pp. 3004-3010, 2010.

[13] J. Li, D.-B. Le, P. P. Ferguson, and J. R. Dahn, "Lithium polyacrylate as a binder for tin-cobalt-carbon negative electrodes in lithium-ion batteries," Electrochimica Acta, vol. 55, no. 8, pp. 2991-2995, 2010.

[14] N. S. Hochgatterer, M. R. Schweiger, S. Koller et al., "Silicon/graphite composite electrodes for high-capacity anodes: influence of binder chemistry on cycling stability," Electrochemical and Solid-State Letters, vol. 11, no. 5, pp. A76-A80, 2008.

[15] S. Komaba, N. Yabuuchi, T. Ozeki et al., "Comparative study of sodium polyacrylate and poly(vinylidene fluoride) as binders for high capacity si-graphite composite negative electrodes in Li-ion batteries," Journal of Physical Chemistry C, vol. 116, no. 1, pp. 1380-1389, 2012.

[16] X. W. Zhou, N. R. Moody, R. E. Jones, J. A. Zimmerman, and E. D. Reedy, "Molecular-dynamics-based cohesive zone law for brittle interfacial fracture under mixed loading conditions: effects of elastic constant mismatch," Acta Materialia, vol. 57, no. 16, pp. 4671-4686, 2009.

[17] X. W. Zhou, J. A. Zimmerman, E. D. Reedy Jr., and N. R. Moody, "Molecular dynamics simulation based cohesive surface representation of mixed mode fracture," Mechanics of Materials, vol. 40, no. 10, pp. 832-845, 2008.

[18] C. R. Dandekar and Y. C. Shin, "Molecular dynamics based cohesive zone law for describing Al-SiC interface mechanics," Composites A: Applied Science and Manufacturing, vol. 42, no. 4, pp. 355-363, 2011.

[19] C. R. Dandekar and Y. C. Shin, "Effect of porosity on the interface behavior of an $\mathrm{Al}_{2} \mathrm{O}_{3}$-aluminum composite: a molecular dynamics study," Composites Science and Technology, vol. 71, no. 3, pp. 350-356, 2011.

[20] Y. Zhang, X. Zhuang, J. Muthu et al., "Load transfer of graphene/carbon nanotube/polyethylene hybrid nanocomposite by molecular dynamics simulation," Composites Part B: Engineering, vol. 63, pp. 27-33, 2014.

[21] Y. Li and G. D. Seidel, "Multiscale modeling of the effects of nanoscale load transfer on the effective elastic properties of unfunctionalized carbon nanotube-polyethylene nanocomposites," Modelling and Simulation in Materials Science and Engineering, vol. 22, no. 2, Article ID 025023, 2014.

[22] A. P. Awasthi, D. C. Lagoudas, and D. C. Hammerand, "Modeling of graphene-polymer interfacial mechanical behavior using molecular dynamics," Modelling and Simulation in Materials Science and Engineering, vol. 17, no. 1, Article ID 015002, 2009.

[23] S. Lee, J. Park, J. Yang, and W. Lu, "Molecular dynamics simulations of the traction-separation response at the interface between PVDF binder and graphite in the electrode of Li-Ion batteries," Journal of the Electrochemical Society, vol. 161, no. 9, pp. A1218-A1223, 2014.

[24] S. M. Foiles, M. I. Baskes, and M. S. Daw, "Embedded-atommethod functions for the fcc metals $\mathrm{Cu}, \mathrm{Ag}, \mathrm{Au}, \mathrm{Ni}, \mathrm{Pd}, \mathrm{Pt}$, and their alloys," Physical Review B, vol. 33, article 7983, 1986.

[25] O. G. Byutner and G. D. Smith, "Quantum chemistry based force field for simulations of poly(vinylidene fluoride)," Macromolecules, vol. 33, no. 11, pp. 4264-4270, 2000.

[26] K. Y. Wong, Molecular design of thiol based nano network for copper-epoxy interfacial adhesion [Ph.D. thesis], The Hong Kong University of Science and Technology, 2008. 
[27] S. Yang, F. Gao, and J. Qu, "A molecular dynamics study of tensile strength between a highly-crosslinked epoxy molding compound and a copper substrate," Polymer, vol. 54, no. 18, pp. 5064-5074, 2013.

[28] Y. Zhang, J. Zhao, N. Wei, J. Jiang, Y. Gong, and T. Rabczuk, "Effects of the dispersion of polymer wrapped two neighbouring single walled carbon nanotubes (SWNTs) on nanoengineering load transfer," Composites Part B: Engineering, vol. 45, no. 1, pp. 1714-1721, 2013.

[29] D. Xin and Q. Han, "Adhesion reliability of the epoxy-cu interface by molecular simulations," The Journal of Adhesion, vol. 91, no. 5, pp. 409-418, 2014.

[30] B. Prathab, V. Subramanian, and T. M. Aminabhavi, "Molecular dynamics simulations to investigate polymer-polymer and polymer-metal oxide interactions," Polymer, vol. 48, no. 1, pp. 409-416, 2007.

[31] S. Kisin, J. Božović Vukić, P. G. T. van der Varst, G. de With, and C. E. Koning, "Estimating the polymer-metal work of adhesion from molecular dynamics simulations," Chemistry of Materials, vol. 19, no. 4, pp. 903-907, 2007.

[32] H. Sun, P. Ren, and J. R. Fried, "The COMPASS force field: parameterization and validation for phosphazenes," Computational and Theoretical Polymer Science, vol. 8, no. 1-2, pp. 229-246, 1998.

[33] P. M. Agrawal, B. M. Rice, and D. L. Thompson, "Predicting trends in rate parameters for self-diffusion on FCC metal surfaces," Surface Science, vol. 515, no. 1, pp. 21-35, 2002.

[34] H. J. Hwang, O.-K. Kwon, and J. W. Kang, "Copper nanocluster diffusion in carbon nanotube," Solid State Communications, vol. 129, no. 11, pp. 687-690, 2004.

[35] S. Lee, J. Park, A. M. Sastry, and W. Lu, "Molecular dynamics simulations of SOC-dependent elasticity of $\mathrm{li}_{x} \mathrm{mn}_{2} \mathrm{o}_{4}$ spinels in Li-ion batteries," Journal of the Electrochemical Society, vol. 160, no. 6, pp. A968-A972, 2013.

[36] A. K. Subramaniyan and C. T. Sun, "Continuum interpretation of virial stress in molecular simulations," International Journal of Solids and Structures, vol. 45, no. 14-15, pp. 4340-4346, 2008.

[37] A. Venkattraman and A. A. Alexeenko, "Direct simulation Monte Carlo modeling of e-beam metal deposition," Journal of Vacuum Science and Technology A, vol. 28, no. 4, pp. 916-924, 2010.

[38] J. Yu and J. G. Amar, "Effects of short-range attraction in metal epitaxial growth," Physical Review Letters, vol. 89, no. 28, Article ID 286103, 2002.

[39] J. Lv, M. Bai, W. Cui, and X. Li, “The molecular dynamic simulation on impact and friction characters of nanofluids with many nanoparticles system," Nanoscale Research Letters, vol. 6, no. 1, article 200, 2011.

[40] Y. Guo and W. Guo, "Structural transformation of partially confined copper nanowires inside defected carbon nanotubes," Nanotechnology, vol. 17, no. 18, 2006. 

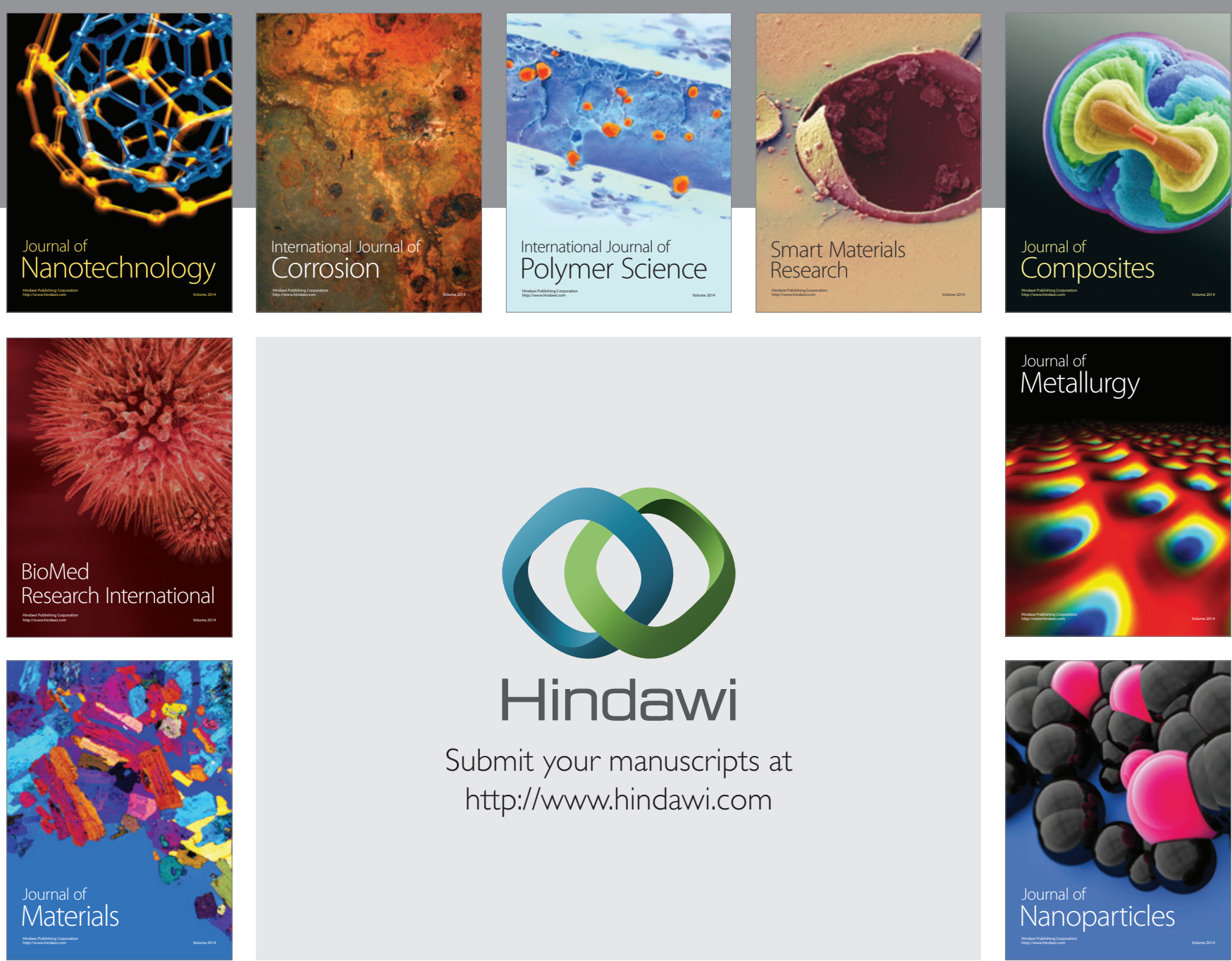

\section{Hindawi}

Submit your manuscripts at

http://www.hindawi.com

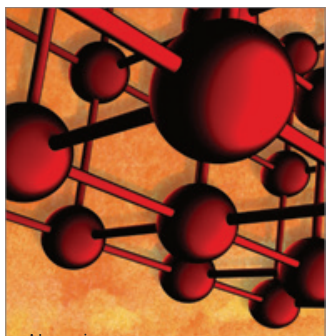

Materials Science and Engineering
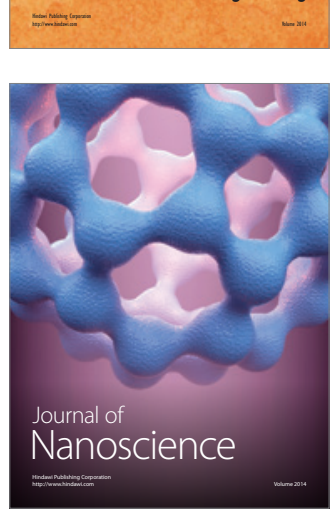
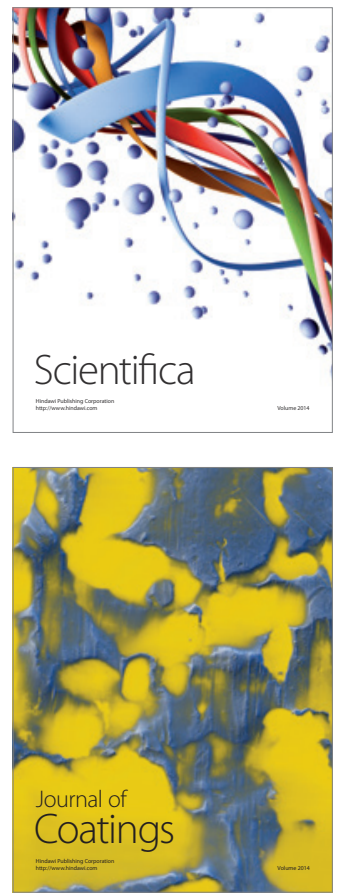
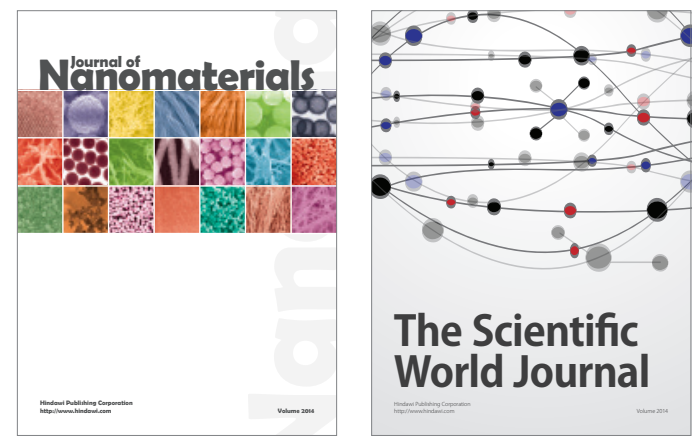

The Scientific World Journal

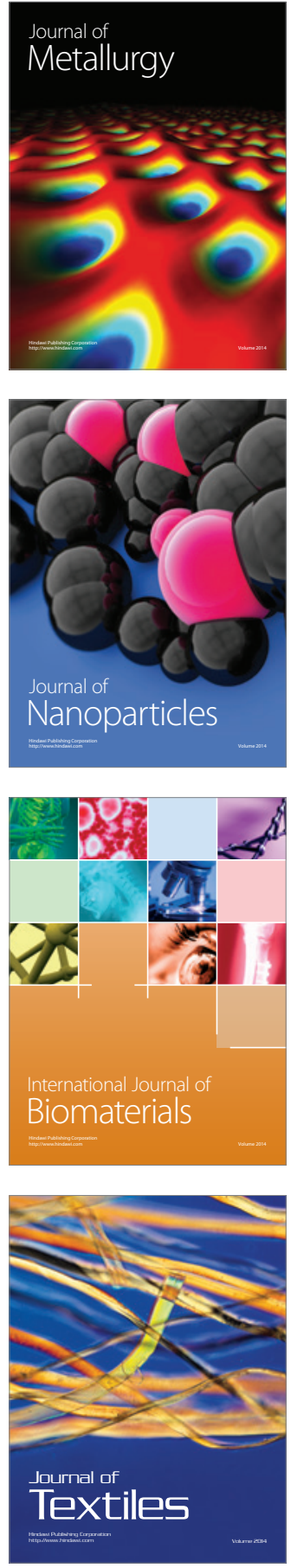